\title{
A Hybrid Extragradient-Like Method for Variational Inequalities, Equilibrium Problems, and an Infinitely Family of Strictly Pseudocontractive Mappings
}

\author{
Yaqin Wang, ${ }^{1,}{ }^{2}$ Hongkun $X u^{3}$ and Xiaoli Fang ${ }^{1}$ \\ ${ }^{1}$ Department of Mathematics, Shaoxing University, Shaoxing 312000, China \\ ${ }^{2}$ Mathematical College, Sichuan University, Sichuan, Chengdu 610064, China \\ ${ }^{3}$ Department of Applied Mathematics, National Sun Yat-sen University, Kaohsiung 80424, Taiwan
}

Correspondence should be addressed to Yaqin Wang, wangyaqin0579@126.com

Received 31 December 2011; Accepted 15 January 2012

Academic Editor: Yonghong Yao

Copyright (C) 2012 Yaqin Wang et al. This is an open access article distributed under the Creative Commons Attribution License, which permits unrestricted use, distribution, and reproduction in any medium, provided the original work is properly cited.

The purpose of this paper is to consider a new scheme by the hybrid extragradient-like method for finding a common element of the set of solutions of a generalized mixed equilibrium problem, the set of solutions of a variational inequality, and the set of fixed points of an infinitely family of strictly pseudocontractive mappings in Hilbert spaces. Then, we obtain a strong convergence theorem of the iterative sequence generated by the proposed iterative algorithm. Our results extend and improve the results of Issara Inchan (2010) and many others.

\section{Introduction}

Let $H$ be a real Hilbert space with inner product $\langle\cdot, \cdot\rangle$ and norm $\|\cdot\|$. Let $C$ be a nonempty closed convex subset of $H$ and $A$ be a mapping of $C$ into $H$. We denote by $F(A)$ the set of fixed points of $A$ and by $P_{C}$ the metric projection of $H$ onto $C$. We also denote by $R$ the set of all real numbers.

Recall the following definitions.

(i) $A$ is called monotone if

$$
\langle A x-A y, x-y\rangle \geq 0, \quad \forall x, y \in C
$$


(ii) $A$ is called $\alpha$-inverse-strongly monotone if there exists a positive constant $\alpha$ such that

$$
\langle A x-A y, x-y\rangle \geq \alpha\|A x-A y\|^{2}, \quad \forall x, y \in C .
$$

(iii) $A$ is called $k$-Lipschitz continuous if there exists a positive constant $k$ such that

$$
\|A x-A y\| \leq k\|x-y\|, \quad \forall x, y \in C
$$

Clearly, every inverse strongly monotone mapping is Lipschitz continuous and monotone.

A mapping $T: C \rightarrow C$ is said to be $\xi$-strictly pseudocontractive if there exists a constant $\xi \in[0,1)$ such that

$$
\|T x-T y\|^{2} \leq\|x-y\|^{2}+\xi\|(I-T) x-(I-T) y\|^{2}, \quad \forall x, y \in C .
$$

It is known that if $T$ is a 0 -strictly pseudocontractive mapping, then $T$ is a nonexpansive mapping. So the class of $\xi$-strictly pseudocontractive mappings includes the class of nonexpansive mappings. such that

Let $\Theta: C \times C \rightarrow R$ be a bifunction. The equilibrium problem for $\Theta$ is to find that $x \in C$

$$
\Theta(x, y) \geq 0, \quad \forall y \in C
$$

The set of solutions of problem (1.5) is denoted by EP.

Given a mapping $A: C \rightarrow H$, let $\Theta(x, y)=\langle A x, y-x\rangle$ for all $x, y \in C$. Then problem (1.5) reduces to the following classical variational inequality problem of finding $x \in C$ such that

$$
\langle A x, y-x\rangle \geq 0, \quad \forall y \in C
$$

The set of solutions of problem (1.6) is denoted by $\operatorname{VI}(C, A)$.

Numerous problems in physics, optimization, saddle point problems, complementarity problems, mechanics, and economics reduce to find a solution of problem (1.5). Many methods have been proposed to solve problem (1.5); see, for instant, [1-3]. In 1997, Combettes and Hirstoaga [4] introduced an iterative scheme of finding the best approximation to initial data when EP is nonempty and proved a strong convergence theorem.

Recently, Peng and Yao [5] introduced the following generalized mixed equilibrium problem of finding $x \in C$ such that

$$
\Theta(x, y)+\varphi(y)-\varphi(x)+\langle B x, y-x\rangle \geq 0, \quad \forall y \in C,
$$

where $B: C \rightarrow H$ is a nonlinear mapping, and $\varphi: C \rightarrow R$ is a function. The set of solutions of problem (1.7) is denoted by G MEP. 
In the case of $B=0$ and $\varphi=0$, then problem (1.7) reduces to problem (1.5). In the case of $\Theta=0, \varphi=0$, and $B=A$, then problem (1.7) reduces to problem (1.6). In the case of $\varphi=0$, problem (1.7) reduces to the generalized equilibrium problem. In the case of $B=0$, problem (1.7) reduces to the following mixed equilibrium problem of finding $x \in C$ such that

$$
\Theta(x, y)+\varphi(y)-\varphi(x) \geq 0, \quad \forall y \in C
$$

which was considered by Ceng and Yao [6]. The set of sulutions of this problem is denoted by MEP.

The problem (1.7) is very general in the sense that it includes, as special cases, some optimization, variational inequalities, minimax problems, the Nash equilibrium problem in noncooperative games, economics, and others (see, for instant, [6])

Recently, S. Takahashi and W. Takahashi [7] introduced the following iteration process:

$$
\begin{gathered}
x_{1}=u \in C, \\
\Theta\left(u_{n}, y\right)+\left\langle A x_{n}, y-u_{n}\right\rangle+\frac{1}{r}\left\langle y-u_{n}, u_{n}-x_{n}\right\rangle \geq 0, \quad \forall y \in C, \\
x_{n+1}=\beta_{n} x_{n}+\left(1-\beta_{n}\right) S\left[\alpha_{n} u+\left(1-\alpha_{n}\right) u_{n}\right], \quad n \geq 1,
\end{gathered}
$$

and used this iteration process to find a common element of the set of fixed points of a nonexpansive mapping $S$ and the set of solutions of a generalized equilibrium problem in a Hilbert space.

In 2008, Bnouhachem et al. [8] introduced the following new extragradient iterative method. Let $C$ be a closed convex subset of a real Hilbert $H, A$ be an $\alpha$-inverse strongly monotone mapping of $C$ into $H$, and let $S$ be a nonexpansive mapping of $C$ into itself such that $F(S) \cap \operatorname{VI}(C, A) \neq \emptyset$. Let the sequences $\left\{x_{n}\right\},\left\{y_{n}\right\}$ be given by

$$
\begin{gathered}
x_{1}, u \in C \text { chosen arbitrary, } \\
y_{n}=P_{C}\left(x_{n}-\lambda_{n} A x_{n}\right), \\
x_{n+1}=\beta_{n} x_{n}+\left(1-\beta_{n}\right) S\left(\alpha_{n} u+\left(1-\alpha_{n}\right) P_{C}\left(x_{n}-\lambda_{n} A y_{n}\right)\right), \quad \forall n \geq 1,
\end{gathered}
$$

where $\left\{\alpha_{n}\right\},\left\{\beta_{n}\right\}$, and $\left\{\lambda_{n}\right\} \subset(0,1)$ satisfy some parameters controlling conditions. They proved that the sequence $\left\{x_{n}\right\}$ converges strongly to a common element of $F(S) \cap \operatorname{VI}(C, A)$.

In 2010, Ceng et al. [9] introduced the following hybrid extragradient-like method. Let $C$ be a nonempty closed convex subset of a real Hilbert space $H, A: C \rightarrow H$ be a monotone, $k$-Lipschitz continuous mapping, and let $S: C \rightarrow C$ be a nonexpansive mapping such that $F(S) \cap \operatorname{VI}(C, A) \neq \emptyset$. Let the sequences $\left\{x_{n}\right\},\left\{y_{n}\right\}$, and $\left\{z_{n}\right\}$ be defined by

$$
\begin{gathered}
x_{0} \in C, \\
y_{n}=\left(1-\gamma_{n}\right) x_{n}+\gamma_{n} P_{C}\left(x_{n}-\lambda_{n} A x_{n}\right),
\end{gathered}
$$




$$
\begin{gathered}
z_{n}=\left(1-\alpha_{n}-\beta_{n}\right) x_{n}+\alpha_{n} y_{n}+\beta_{n} S P_{C}\left(x_{n}-\lambda_{n} A y_{n}\right), \\
C_{n}=\left\{z \in C:\left\|z_{n}-z\right\|^{2} \leq\left\|x_{n}-z\right\|^{2}+\left(3-3 \gamma_{n}+\alpha_{n}\right) b^{2}\left\|A x_{n}\right\|^{2}\right\} \\
Q_{n}=\left\{z \in C,\left\langle x_{n}-z, x_{0}-x_{n}\right\rangle \geq 0\right\} \\
x_{n+1}=P_{C_{n} \cap Q_{n}} x_{0}, \quad \forall n \geq 0 .
\end{gathered}
$$

Under the suitable conditions, they proved the sequences $\left\{x_{n}\right\},\left\{y_{n}\right\},\left\{z_{n}\right\}$ converge strongly to the same point $P_{F(S) \cap V I(C, A)} x_{0}$.

In 2010, Inchan [10] introduced a new iterative scheme by the hybrid extragradient method in a Hilbert space $H$ as follows: $x_{0} \in H, C_{1}=C \subset H, x_{1}=P_{C} x_{0}$, and let

$$
\begin{gathered}
u_{n} \in C, \quad \Theta\left(u_{n}, y\right)+\left\langle B x_{n}, y-u_{n}\right\rangle+\frac{1}{r_{n}}\left\langle y-u_{n}, u_{n}-x_{n}\right\rangle \geq 0, \quad \forall y \in C, \\
y_{n}=P_{C}\left(u_{n}-\lambda_{n} A u_{n}\right), \\
z_{n}=\alpha_{n} x_{n}+\left(1-\alpha_{n}\right) S\left(\beta_{n} x_{n}+\left(1-\beta_{n}\right) P_{C}\left(u_{n}-\lambda_{n} A y_{n}\right)\right), \\
C_{n+1}=\left\{z \in C_{n}:\left\|z_{n}-z\right\| \leq\left\|x_{n}-z\right\|\right\}, \\
x_{n+1}=P_{C_{n+1}} x_{0}, \quad \forall n \geq 0,
\end{gathered}
$$

where $\left\{\alpha_{n}\right\},\left\{\beta_{n}\right\}$, and $\left\{\lambda_{n}\right\} \subset(0,1)$ satisfy some parameters controlling conditions. They proved that $\left\{x_{n}\right\}$ and $\left\{u_{n}\right\}$ strongly converge to the same common element of the set of fixed points of a nonexpansive mapping, the set of solutions of an equilibrium problem, and the set of solutions of the variational inequality for nonexpansive mappings.

Very recently, Wang [11] defined the mapping $W_{n}$ as follows:

$$
\begin{aligned}
& U_{n, n+1}=I, \\
& U_{n, n}=\gamma_{n} T_{n}^{\prime} U_{n, n+1}+\left(1-\gamma_{n}\right) I, \\
& U_{n, n-1}=\gamma_{n-1} T_{n-1}^{\prime} U_{n, n}+\left(1-\gamma_{n-1}\right) I, \\
& \vdots \\
& U_{n, k}=\gamma_{k} T_{k}^{\prime} U_{n, k+1}+\left(1-\gamma_{k}\right) I, \\
& U_{n, k-1}=\gamma_{k-1} T_{k-1}^{\prime} U_{n, k}+\left(1-\gamma_{k-1}\right) I, \\
& \vdots \\
& U_{n, 2}=\gamma_{2} T_{2}^{\prime} U_{n, 3}+\left(1-\gamma_{2}\right) I, \\
& W_{n}=U_{n, 1}=\gamma_{1} T_{1}^{\prime} U_{n, 2}+\left(1-\gamma_{1}\right) I,
\end{aligned}
$$

where $\gamma_{1}, \gamma_{2}, \ldots$ are real numbers such that $0 \leq \gamma_{n} \leq 1, T_{i}^{\prime}=\theta_{i} I+\left(1-\theta_{i}\right) T_{i}$, where $T_{i}$ is a $\mu_{i^{-}}$ strictly pseudocontractive mapping of $C$ into itself and $\theta_{i} \in\left[\mu_{i}, 1\right)$. It follows from [12] that $T_{i}^{\prime}$ is nonexpansive and $F\left(T_{i}\right)=F\left(T_{i}^{\prime}\right)$. Nonexpansivity of each $T_{i}^{\prime}$ ensures the nonexpansivity of $W_{n}$. 
Motivated and inspired by the above work, in this paper, we introduced the following new iterative scheme by the extragradient-like method: $C_{1}=C \subset H, x_{1}=P_{C} x_{0}$,

$$
\begin{gathered}
u_{n} \in C \text { such that } \Theta\left(u_{n}, y\right)+\left\langle B x_{n}, y-u_{n}\right\rangle+\varphi(y)-\varphi\left(u_{n}\right)+\frac{1}{r_{n}}\left\langle y-u_{n}, u_{n}-x_{n}\right\rangle \geq 0, \quad y \in C, \\
y_{n}=\left(1-s_{n}\right) u_{n}+s_{n} P_{C}\left(u_{n}-\lambda_{n} A u_{n}\right), \\
z_{n}=\alpha_{n} x_{n}+\left(1-\alpha_{n}\right) W_{n}\left(\beta_{n} x_{n}+\left(1-\beta_{n}\right) P_{C}\left(u_{n}-\lambda_{n} A y_{n}\right)\right), \\
C_{n+1}=\left\{z \in C_{n}:\left\|z_{n}-z\right\|^{2} \leq\left\|x_{n}-z\right\|^{2}+3\left(1-s_{n}\right) b^{2}\left\|A u_{n}\right\|^{2}\right\}, \\
x_{n+1}=P_{C_{n+1}} x_{0}, \quad \forall n \geq 0,
\end{gathered}
$$

where $A, B: C \rightarrow H$ and $A$ is a monotone, $k$-Lipschitz continuous mapping, and $B$ is a $\beta$-inverse strongly monotone mapping. Then under the suitable conditions, we derive some strong convergence results.

\section{Preliminaries}

Let $C$ be a nonempty closed and convex subset of a Hilbert space $H$, for any $x \in H$, and there exists a unique nearest point in $C$, denoted by $P_{C} x$ such that

$$
\left\|x-P_{C} x\right\| \leq\|x-y\|, \quad \forall y \in C
$$

The projection operator $P_{C}: H \rightarrow C$ is nonexpansive. Moreover, $P_{C} x$ is characterized by the following properties: for every $x \in H$ and $y \in C$,

$$
\begin{gathered}
\|x-y\|^{2} \geq\left\|x-P_{C} x\right\|^{2}+\left\|y-P_{C} x\right\|^{2} \\
\left\langle x-P_{C} x, y-P_{C} x\right\rangle \leq 0 .
\end{gathered}
$$

Suppose that $A$ is monotone and continuous. Then the solutions of the variational inequality $\operatorname{VI}(C, A)$ can be characterized as solutions of the so-called Minty variational inequality:

$$
x^{*} \in \mathrm{VI}(C, A) \Longleftrightarrow\left\langle A x, x-x^{*}\right\rangle \geq 0, \quad \forall x \in C
$$

In what follows, we shall make use of the following lemmas.

Lemma 2.1. Let $H$ be a real Hilbert space. Then for any $x, y \in H$, we have

(i) $\|x \pm y\|^{2}=\|x\|^{2} \pm 2\langle x, y\rangle+\|y\|^{2}$,

(ii) $\|t x+(1-t) y\|^{2}=t\|x\|^{2}+(1-t)\|y\|^{2}-t(1-t)\|x-y\|^{2}$, for all $t \in[0,1]$.

We denote by $N_{C}(v)$ the normal cone for $C$ at a point $v \in C$, that is $N_{C}(v):=\left\{x^{*} \in E^{*}\right.$ : $\left\langle v-y, x^{*}\right\rangle \geq 0$ for all $\left.y \in C\right\}$. In the following, we shall use the following Lemma. 
Lemma 2.2 (see [13]). Let $C$ be a nonempty closed convex subset of a Banach space $E$, and let $A$ be a monotone and hemicontinuous operator of $C$ into $E^{*}$. Let $T \subset E \times E^{*}$ be an operator defined as follows:

$$
T v= \begin{cases}A v+N_{C}(v), & v \in C, \\ \emptyset, & v \notin C .\end{cases}
$$

Then $T$ is maximal monotone, and $T^{-1} 0=\mathrm{VI}(C, A)$.

Lemma 2.3 (see [14]). Let $C$ be a nonempty closed convex subset of a strictly convex Banach space E. Let $T_{1}^{\prime}, T_{2}^{\prime}, \ldots$ be nonexpansive mappings of $C$ into itself such that $\bigcap_{i=1}^{\infty} F\left(T_{i}^{\prime}\right) \neq \emptyset$ and $\gamma_{1}, \gamma_{2}, \ldots$ be real numbers such that $0<\gamma_{i} \leq b<1$ for every $i=1,2, \ldots$. Then for any $x \in C$ and $k \in N$, the limit $\lim _{n \rightarrow \infty} U_{n, k}$ exists.

Using Lemma 2.3, define the mapping $W$ of $C$ into itself as follows:

$$
W x:=\lim _{n \rightarrow \infty} W_{n} x=\lim _{n \rightarrow \infty} U_{n, 1} x, \quad x \in C .
$$

Such a mapping $W$ is called the modified $W$ mapping generalized by $T_{1}, T_{2}, \ldots, \gamma_{1}, \gamma_{2}, \ldots$, and $\theta_{1}, \theta_{2}, \ldots$

Lemma 2.4 (see [14]). Let $C$ be a nonempty closed convex subset of a strictly convex Banach space E. Let $T_{1}^{\prime}, T_{2}^{\prime}, \ldots$ be nonexpansive mappings of $C$ into itself such that $\bigcap_{i=1}^{\infty} F\left(T_{i}^{\prime}\right) \neq \emptyset$ and $\gamma_{1}, \gamma_{2}, \ldots$ be real numbers such that $0<\gamma_{i} \leq b<1$ for every $i=1,2, \ldots$. Then $W$ is a nonexpansive mapping satisfying that $F(W)=\bigcap_{i=1}^{\infty} F\left(T_{i}^{\prime}\right)$.

Lemma 2.5 (see [15]). Let $C$ be a nonempty closed convex subset of a strictly convex Banach space E. Let $T_{1}^{\prime}, T_{2}^{\prime}, \ldots$ be nonexpansive mappings of $C$ into itself such that $\bigcap_{i=1}^{\infty} F\left(T_{i}^{\prime}\right) \neq \emptyset$ and $\gamma_{1}, \gamma_{2}, \ldots$ be real numbers such that $0<\gamma_{i} \leq b<1$ for every $i=1,2, \ldots$. If $K$ is any bounded subset of $C$, then

$$
\limsup _{n \rightarrow \infty}\left\|W x-W_{n} x\right\|=0
$$

For solving the equilibrium problem, let us assume that $\Theta$ satisfies the following conditions:

(H1) $\Theta(x, x)=0$ for all $x \in C$,

(H2) $\Theta$ is monotone, that is, $\Theta(x, y)+\Theta(y, x) \leq 0$ for all $x, y \in C$,

(H3) for each $y \in C, x \mapsto \Theta(x, y)$ is weakly upper semicontinuous,

(H4) for each $x \in C, y \mapsto \Theta(x, y)$ is convex and lower semicontinuous,

(A1) for each $x \in H$ and $r>0$, there exists a bounded subset $D_{x} \subset C$ and $y_{x} \in C$ such that for any $z \in C \backslash D_{x}$,

$$
\Theta\left(z, y_{x}\right)+\varphi\left(y_{x}\right)-\varphi(z)+\frac{1}{r}\left\langle y_{x}-z, z-x\right\rangle<0,
$$

(A2) $C$ is a bounded set. 
Lemma 2.6 (see [6]). Let $C$ be a closed subset of $H$. Let $\varphi: C \rightarrow R$ be a lower semicontinuous and convex function, and $\Theta$ be a bifunction from $C \times C$ to R satisfying (H1)-(H4). For $r>0$ and $x \in H$, define a mapping $T_{r}: H \rightarrow C$ as follows:

$$
T_{r}(x)=\left\{z \in C: \Theta(z, y)+\varphi(y)-\varphi(z)+\frac{1}{r}\langle y-z, z-x\rangle \geq 0, \forall y \in C\right\}
$$

for all $x \in H$. Assume that either (A1) or (A2) holds. Then the following results hold:

(1) $T_{r}(x) \neq \emptyset$ for each $x \in H$, and $T_{r}$ is single valued,

(2) $T_{r}$ is firmly nonexpansive, that is, for all $x, y \in H,\left\|T_{r} x-T_{r} y\right\|^{2} \leq\left\langle T_{r} x-T_{r} y, x-y\right\rangle$,

(3) $F\left(T_{r}\right)=\mathrm{MEP}$,

(4) MEP is closed and convex.

\section{Strong Convergence Theorems}

Theorem 3.1. Let $C$ be a nonempty closed convex subset of a real Hilbert space $H$. Let $\Theta$ be a bifunction from $C \times C$ into $R$ satisfying (H1)-(H4) and $\varphi: C \rightarrow R$ be a lower semicontinuous and convex function with (A1) or (A2). Let $A: C \rightarrow H$ be a monotone, $k$-Lipschitz continuous mapping and $B: C \rightarrow H$ be a $\beta$-inverse-strongly monotone mapping. Let $T_{i}: C \rightarrow C$ be a $\mu_{i^{-}}$ strictly pseudocontractive mapping with $F=: \bigcap_{i=1}^{\infty} F\left(T_{i}\right) \cap \operatorname{VI}(C, A) \cap G \mathrm{MEP} \neq \emptyset$ and $\left\{\gamma_{i}\right\}$ be a real sequence such that $0<\gamma_{i} \leq b<1$, for all $i \geq 1$. Assume that the control sequences $\left\{\alpha_{n}\right\},\left\{\beta_{n}\right\}$, $\left\{s_{n}\right\} \subset[0,1],\left\{r_{n}\right\} \subset(0,2 \beta)$, and $\left\{\lambda_{n}\right\} \subset(0,1 / 2 k)$ satisfy the following conditions:

(i) $\lim \sup _{n \rightarrow \infty} \alpha_{n}<1, \limsup _{n \rightarrow \infty} \beta_{n}<1$,

(ii) $0<a \leq \lambda_{n} \leq b<1 / 2 k, 0<d \leq r_{n} \leq e<2 \beta$,

(iii) $s_{n} \rightarrow 1(n \rightarrow \infty)$ and $s_{n}>3 / 4$ for all $n \geq 0$.

Then the sequence $\left\{x_{n}\right\}$ defined by (1.14) converges strongly to $P_{F} x_{0}$.

Proof. We divide the proof into several steps.

Step 1 ( $\left\{x_{n}\right\}$ is well defined). Indeed, for any $q \in F$. Put $t_{n}=P_{C}\left(u_{n}-\lambda_{n} A y_{n}\right)$. Since $u_{n}=$ $T_{r_{n}}\left(x_{n}-r_{n} B x_{n}\right), q=T_{r_{n}}\left(q-r_{n} B q\right)$, and $B$ is $\beta$-inverse-strongly monotone and $r_{n} \in[0,2 \beta]$, for any $n \geq 0$, we have

$$
\begin{aligned}
\left\|u_{n}-q\right\|^{2} & =\left\|T_{r_{n}}\left(x_{n}-r_{n} B x_{n}\right)-T_{r_{n}}\left(q-r_{n} B q\right)\right\|^{2} \\
& \leq\left\|x_{n}-r_{n} B x_{n}-\left(q-r_{n} B q\right)\right\|^{2} \\
& \leq\left\|x_{n}-q\right\|^{2}-2 r_{n}\left\langle B x_{n}-B q, x_{n}-q\right\rangle+r_{n}^{2}\left\|B x_{n}-B q\right\|^{2} \\
& \leq\left\|x_{n}-q\right\|^{2}+r_{n}\left(r_{n}-2 \beta\right)\left\|B x_{n}-B q\right\|^{2} \\
& \leq\left\|x_{n}-q\right\|^{2} .
\end{aligned}
$$


It follows from (2.2) and (2.4) that

$$
\begin{aligned}
\left\|t_{n}-q\right\|^{2} \leq & \left\|u_{n}-\lambda_{n} A y_{n}-q\right\|^{2}-\left\|u_{n}-\lambda_{n} A y_{n}-t_{n}\right\|^{2} \\
= & \left\|u_{n}-q\right\|^{2}-\left\|u_{n}-t_{n}\right\|^{2}-2 \lambda_{n}\left\langle A y_{n}, t_{n}-q\right\rangle \\
= & \left\|u_{n}-q\right\|^{2}-\left\|u_{n}-y_{n}\right\|^{2}-\left\|y_{n}-t_{n}\right\|^{2}-2 \lambda_{n}\left\langle A y_{n}, y_{n}-q\right\rangle \\
& -2\left\langle u_{n}-y_{n}, y_{n}-t_{n}\right\rangle+2 \lambda_{n}\left\langle A y_{n}, y_{n}-t_{n}\right\rangle \\
\leq & \left\|u_{n}-q\right\|^{2}-\left\|u_{n}-y_{n}\right\|^{2}-\left\|y_{n}-t_{n}\right\|^{2}+2\left\langle u_{n}-y_{n}-\lambda_{n} A y_{n}, t_{n}-y_{n}\right\rangle .
\end{aligned}
$$

In addition, we have

$$
\begin{aligned}
\left\langle u_{n}-y_{n}-\lambda_{n} A y_{n}, t_{n}-y_{n}\right\rangle= & \left\langle u_{n}-y_{n}-\lambda_{n} A u_{n}, t_{n}-y_{n}\right\rangle+\lambda_{n}\left\langle A u_{n}-A y_{n}, t_{n}-y_{n}\right\rangle \\
\leq & s_{n}\left\langle u_{n}-\lambda_{n} A u_{n}-P_{C}\left(u_{n}-\lambda_{n} A u_{n}\right), t_{n}-y_{n}\right\rangle \\
& +\lambda_{n}\left(s_{n}-1\right)\left\langle A u_{n}, t_{n}-y_{n}\right\rangle+\lambda_{n} k\left\|u_{n}-y_{n}\right\|\left\|t_{n}-y_{n}\right\|
\end{aligned}
$$

and by (2.3), we obtain

$$
\begin{aligned}
\left\langle u_{n}-\right. & \left.\lambda_{n} A u_{n}-P_{C}\left(u_{n}-\lambda_{n} A u_{n}\right), t_{n}-y_{n}\right\rangle \\
= & \left\langle u_{n}-\lambda_{n} A u_{n}-P_{C}\left(u_{n}-\lambda_{n} A u_{n}\right),\left(1-s_{n}\right)\left(t_{n}-u_{n}\right)+s_{n}\left(t_{n}-P_{C}\left(u_{n}-\lambda_{n} A u_{n}\right)\right)\right\rangle \\
\leq & \left(1-s_{n}\right) \lambda_{n}\left\|A u_{n}\right\|\left(\left\|t_{n}-y_{n}\right\|+\left\|y_{n}-u_{n}\right\|\right) \\
& +s_{n}\left\langle u_{n}-\lambda_{n} A u_{n}-P_{C}\left(u_{n}-\lambda_{n} A u_{n}\right), t_{n}-P_{C}\left(u_{n}-\lambda_{n} A u_{n}\right)\right\rangle \\
\leq & \left(1-s_{n}\right) \lambda_{n}\left\|A u_{n}\right\|\left(\left\|t_{n}-y_{n}\right\|+\left\|y_{n}-u_{n}\right\|\right) .
\end{aligned}
$$

It follows from (3.2)-(3.4) that

$$
\begin{aligned}
\left\|t_{n}-q\right\|^{2} \leq & \left\|u_{n}-q\right\|^{2}-\left\|u_{n}-y_{n}\right\|^{2}-\left\|y_{n}-t_{n}\right\|^{2}+2 s_{n}\left(1-s_{n}\right) \lambda_{n}\left\|A u_{n}\right\|\left(\left\|t_{n}-y_{n}\right\|+\left\|y_{n}-u_{n}\right\|\right) \\
& +2 \lambda_{n}\left(1-s_{n}\right)\left\|A u_{n}\right\|\left\|t_{n}-y_{n}\right\|+2 \lambda_{n} k\left\|u_{n}-y_{n}\right\|\left\|t_{n}-y_{n}\right\| \\
\leq & \left\|u_{n}-q\right\|^{2}-\left\|u_{n}-y_{n}\right\|^{2}-\left\|y_{n}-t_{n}\right\|^{2} \\
& +s_{n}\left(1-s_{n}\right)\left[2 b^{2}\left\|A u_{n}\right\|^{2}+\left\|t_{n}-y_{n}\right\|^{2}+\left\|y_{n}-u_{n}\right\|^{2}\right] \\
& +\left(1-s_{n}\right)\left[b^{2}\left\|A u_{n}\right\|^{2}+\left\|t_{n}-y_{n}\right\|^{2}\right]+k b\left[\left\|t_{n}-y_{n}\right\|^{2}+\left\|y_{n}-u_{n}\right\|^{2}\right] \\
= & \left\|u_{n}-q\right\|^{2}-\left(s_{n}-b k\right)\left\|u_{n}-y_{n}\right\|^{2}-\left(2 s_{n}-1-k b\right)\left\|t_{n}-y_{n}\right\|^{2}+3\left(1-s_{n}\right) b^{2}\left\|A u_{n}\right\|^{2} \\
\leq & \left\|u_{n}-q\right\|^{2}+3\left(1-s_{n}\right) b^{2}\left\|A u_{n}\right\|^{2} .
\end{aligned}
$$


Setting $w_{n}=\beta_{n} x_{n}+\left(1-\beta_{n}\right) t_{n}$. Therefore, from (1.14), (3.1), and (3.5), we get the following:

$$
\begin{aligned}
\left\|z_{n}-q\right\|^{2} & \leq \alpha_{n}\left\|x_{n}-q\right\|^{2}+\left(1-\alpha_{n}\right)\left\|W_{n} w_{n}-q\right\|^{2} \\
& \leq \alpha_{n}\left\|x_{n}-q\right\|^{2}+\left(1-\alpha_{n}\right)\left\|w_{n}-q\right\|^{2} \\
& \leq \alpha_{n}\left\|x_{n}-q\right\|^{2}+\left(1-\alpha_{n}\right) \beta_{n}\left\|x_{n}-q\right\|^{2}+\left(1-\alpha_{n}\right)\left(1-\beta_{n}\right)\left\|t_{n}-q\right\|^{2} \\
& \leq\left(\alpha_{n}+\left(1-\alpha_{n}\right) \beta_{n}+\left(1-\alpha_{n}\right)\left(1-\beta_{n}\right)\right)\left\|x_{n}-q\right\|^{2}+3\left(1-\alpha_{n}\right)\left(1-\beta_{n}\right)\left(1-s_{n}\right) b^{2}\left\|A u_{n}\right\|^{2} \\
& \leq\left\|x_{n}-q\right\|^{2}+3\left(1-s_{n}\right) b^{2}\left\|A u_{n}\right\|^{2} .
\end{aligned}
$$

So, $q \in C_{n}$ and hence $F \subset C_{n}$ for all $n \geq 1$. It is easy to see that $C_{n}$ is closed and convex for all $n \geq 1$. This implies that $\left\{x_{n}\right\}$ and $\left\{u_{n}\right\}$ are well defined.

Step 2 ( $\left\{x_{n}\right\}$ is a Cauchy sequence). It is easy to see that $F$ is closed and convex. From $x_{n+1}=$ $P_{C_{n+1}} x_{0} \in C_{n+1} \subset C_{n}$ and $x_{n}=P_{C_{n}} x_{0}$, for any $q \in F$, we have

$$
\left\|x_{n}-x_{0}\right\| \leq\left\|x_{n+1}-x_{0}\right\| \leq\left\|q-x_{0}\right\| \text {. }
$$

So $\left\{x_{n}\right\}$ is bounded, and $\lim _{n \rightarrow \infty}\left\|x_{n}-x_{0}\right\|$ exists. So it follows from (3.1), (3.6), and the continuity of $A$ that $\left\{u_{n}\right\},\left\{z_{n}\right\}$, and $\left\{A u_{n}\right\}$ are bounded. By the construction of $C_{n}$, we have $C_{m} \subset C_{n}$ and $x_{m}=P_{C_{m}} x_{0} \in C_{n}$ for any positive integer $m \geq n$. So from (2.2), we have

$$
\left\|x_{m}-x_{n}\right\|^{2} \leq\left\|x_{m}-x_{0}\right\|^{2}-\left\|x_{n}-x_{0}\right\|^{2} .
$$

Letting $m, n \rightarrow \infty$ in (3.8), we have $\left\|x_{m}-x_{n}\right\| \rightarrow 0$, which implies that $\left\{x_{n}\right\}$ is a Cauchy sequence. So there exists $z \in C$ such that $x_{n} \rightarrow z(n \rightarrow \infty)$.

Step $3\left(\lim _{n \rightarrow \infty}\left\|w_{n}-W w_{n}\right\|=0\right)$. From (3.8), we have

$$
\lim _{n \rightarrow \infty}\left\|x_{n}-x_{n+1}\right\|=0 \text {. }
$$

Since $x_{n+1} \in C_{n+1}$, by (3.9) and condition (iii), we obtain that

$$
\left\|z_{n}-x_{n+1}\right\| \leq\left\|x_{n}-x_{n+1}\right\|+3\left(1-s_{n}\right) b^{2}\left\|A u_{n}\right\|^{2} \longrightarrow 0 \quad(n \longrightarrow \infty) .
$$

So

$$
\lim _{n \rightarrow \infty}\left\|z_{n}-x_{n}\right\|=0
$$

Since

$$
\left\|z_{n}-x_{n}\right\|=\left(1-\alpha_{n}\right)\left\|x_{n}-W_{n} w_{n}\right\|
$$

from (3.11) and condition (i), we have

$$
\lim _{n \rightarrow \infty}\left\|x_{n}-W_{n} w_{n}\right\|=0
$$


For any $q \in F$, from (3.1) and (3.5), we obtain that

$$
\begin{aligned}
\left\|w_{n}-q\right\|^{2} & \leq \beta_{n}\left\|x_{n}-q\right\|^{2}+\left(1-\beta_{n}\right)\left\|t_{n}-q\right\|^{2} \\
& \leq \beta_{n}\left\|x_{n}-q\right\|^{2}+\left(1-\beta_{n}\right)\left\|u_{n}-q\right\|^{2}+3\left(1-s_{n}\right) b^{2}\left\|A u_{n}\right\|^{2} \\
& \leq\left\|x_{n}-q\right\|^{2}-\left(1-\beta_{n}\right) r_{n}\left(2 \beta-r_{n}\right)\left\|B x_{n}-B q\right\|^{2}+3\left(1-s_{n}\right) b^{2}\left\|A u_{n}\right\|^{2} .
\end{aligned}
$$

Therefore, we have

$$
\begin{aligned}
\left\|z_{n}-q\right\|^{2} & \leq \alpha_{n}\left\|x_{n}-q\right\|^{2}+\left(1-\alpha_{n}\right)\left\|w_{n}-q\right\|^{2} \\
& \leq\left\|x_{n}-q\right\|^{2}-\left(1-\alpha_{n}\right)\left(1-\beta_{n}\right) r_{n}\left(2 \beta-r_{n}\right)\left\|B x_{n}-B q\right\|^{2}+3\left(1-s_{n}\right) b^{2}\left\|A u_{n}\right\|^{2},
\end{aligned}
$$

which implies that

$$
\begin{aligned}
& \left(1-\alpha_{n}\right)\left(1-\beta_{n}\right) r_{n}\left(2 \beta-r_{n}\right)\left\|B x_{n}-B q\right\|^{2} \\
& \quad \leq\left\|x_{n}-q\right\|^{2}-\left\|z_{n}-q\right\|^{2}+3\left(1-s_{n}\right) b^{2}\left\|A u_{n}\right\|^{2} \\
& \quad \leq\left\|z_{n}-x_{n}\right\|\left(\left\|z_{n}-q\right\|+\left\|x_{n}-q\right\|\right)+3\left(1-s_{n}\right) b^{2}\left\|A u_{n}\right\|^{2} .
\end{aligned}
$$

Combining the above inequality, (3.11) and conditions (i)-(iii), we have

$$
\lim _{n \rightarrow \infty}\left\|B x_{n}-B q\right\|=0 .
$$

It follows from Lemma 2.6 that

$$
\begin{aligned}
\left\|u_{n}-q\right\|^{2} & =\left\|T_{r_{n}}\left(x_{n}-r_{n} B x_{n}\right)-T_{r_{n}}\left(q-r_{n} B q\right)\right\|^{2} \\
& \leq\left\langle x_{n}-r_{n} B x_{n}-\left(q-r_{n} B q\right), u_{n}-q\right\rangle \\
& =\frac{1}{2}\left(\left\|x_{n}-r_{n} B x_{n}-\left(q-r_{n} B q\right)\right\|^{2}+\left\|u_{n}-q\right\|^{2}-\left\|x_{n}-r_{n} B x_{n}-\left(q-r_{n} B q\right)-\left(u_{n}-q\right)\right\|^{2}\right) \\
& \leq \frac{1}{2}\left(\left\|x_{n}-q\right\|^{2}+\left\|u_{n}-q\right\|^{2}-\left\|x_{n}-u_{n}\right\|^{2}+2 r_{n}\left\langle x_{n}-u_{n}, B x_{n}-B q\right\rangle-r_{n}^{2}\left\|B x_{n}-B q\right\|^{2}\right) .
\end{aligned}
$$

Therefore,

$$
\left\|u_{n}-q\right\|^{2} \leq\left\|x_{n}-q\right\|^{2}-\left\|x_{n}-u_{n}\right\|^{2}+2 r_{n}\left\langle x_{n}-u_{n}, B x_{n}-B q\right\rangle-r_{n}^{2}\left\|B x_{n}-B q\right\|^{2} .
$$


By (3.5) and (3.19), we have

$$
\begin{aligned}
\left\|z_{n}-q\right\|^{2} \leq & \alpha_{n}\left\|x_{n}-q\right\|^{2}+\left(1-\alpha_{n}\right)\left\|w_{n}-q\right\|^{2} \\
\leq & \alpha_{n}\left\|x_{n}-q\right\|^{2}+\left(1-\alpha_{n}\right)\left(\beta_{n}\left\|x_{n}-q\right\|^{2}+\left(1-\beta_{n}\right)\left\|t_{n}-q\right\|^{2}\right) \\
\leq & \alpha_{n}\left\|x_{n}-q\right\|^{2}+\left(1-\alpha_{n}\right)\left(\beta_{n}\left\|x_{n}-q\right\|^{2}+\left(1-\beta_{n}\right)\left\|u_{n}-q\right\|^{2}+3\left(1-s_{n}\right) b^{2}\left\|A u_{n}\right\|^{2}\right) \\
\leq & \left\|x_{n}-q\right\|^{2}-\left(1-\alpha_{n}\right)\left(1-\beta_{n}\right)\left\|x_{n}-u_{n}\right\|^{2}+2 r_{n}\left(1-\alpha_{n}\right)\left(1-\beta_{n}\right)\left\langle x_{n}-u_{n}, B x_{n}-B q\right\rangle \\
& -\left(1-\alpha_{n}\right)\left(1-\beta_{n}\right) r_{n}^{2}\left\|B x_{n}-B q\right\|^{2}+3\left(1-s_{n}\right) b^{2}\left\|A u_{n}\right\|^{2} .
\end{aligned}
$$

It follows from (3.20) that

$$
\begin{aligned}
\left(1-\alpha_{n}\right)\left(1-\beta_{n}\right)\left\|x_{n}-u_{n}\right\|^{2} \leq & \left\|x_{n}-q\right\|^{2}-\left\|z_{n}-q\right\|^{2}+2 r_{n}\left(1-\alpha_{n}\right)\left(1-\beta_{n}\right)\left\langle x_{n}-u_{n}, B x_{n}-B q\right\rangle \\
& -\left(1-\alpha_{n}\right)\left(1-\beta_{n}\right) r_{n}^{2}\left\|B x_{n}-B q\right\|^{2}+3\left(1-s_{n}\right) b^{2}\left\|A u_{n}\right\|^{2} \\
\leq & \left\|z_{n}-x_{n}\right\|\left(\left\|z_{n}-q\right\|+\left\|x_{n}-q\right\|\right)+2 e\left\|x_{n}-u_{n}\right\|\left\|B x_{n}-B q\right\| \\
& +e^{2}\left\|B x_{n}-B q\right\|^{2}+3\left(1-s_{n}\right) b^{2}\left\|A u_{n}\right\|^{2} .
\end{aligned}
$$

Therefore, from (3.11), (3.17), (3.21), and conditions (i), (iii),

$$
\lim _{n \rightarrow \infty}\left\|x_{n}-u_{n}\right\|=0,
$$

which implies that $u_{n} \rightarrow z(n \rightarrow \infty)$. And from (3.1), (3.5), and (3.6), we have

$$
\begin{aligned}
\left\|z_{n}-q\right\|^{2} \leq & \alpha_{n}\left\|x_{n}-q\right\|^{2}+\left(1-\alpha_{n}\right) \beta_{n}\left\|x_{n}-q\right\|^{2}+\left(1-\alpha_{n}\right)\left(1-\beta_{n}\right)\left\|t_{n}-q\right\|^{2} \\
\leq & \left\|x_{n}-q\right\|^{2}-\left(1-\alpha_{n}\right)\left(1-\beta_{n}\right)\left(s_{n}-b k\right)\left\|u_{n}-y_{n}\right\|^{2} \\
& -\left(1-\alpha_{n}\right)\left(1-\beta_{n}\right)\left(2 s_{n}-1-k b\right)\left\|t_{n}-y_{n}\right\|^{2}+3\left(1-s_{n}\right) b^{2}\left\|A u_{n}\right\|^{2} .
\end{aligned}
$$

Thus it follows that

$$
\begin{aligned}
& \left(1-\alpha_{n}\right)\left(1-\beta_{n}\right)\left(s_{n}-b k\right)\left\|u_{n}-y_{n}\right\|^{2}+\left(1-\alpha_{n}\right)\left(1-\beta_{n}\right)\left(2 s_{n}-1-k b\right)\left\|t_{n}-y_{n}\right\|^{2} \\
& \quad \leq\left\|x_{n}-q\right\|^{2}-\left\|z_{n}-q\right\|^{2}+3\left(1-s_{n}\right) b^{2}\left\|A u_{n}\right\|^{2} \\
& \quad \leq\left\|z_{n}-x_{n}\right\|\left(\left\|z_{n}-q\right\|+\left\|x_{n}-q\right\|\right)+3\left(1-s_{n}\right) b^{2}\left\|A u_{n}\right\|^{2} .
\end{aligned}
$$

Therefore, from (3.11), (3.24) and conditions (i)-(iii), we obtain $\lim _{n \rightarrow \infty}\left\|u_{n}-y_{n}\right\|=0$ and $\lim _{n \rightarrow \infty}\left\|t_{n}-y_{n}\right\|=0$. Furthermore, we have

$$
\lim _{n \rightarrow \infty}\left\|t_{n}-u_{n}\right\|=0
$$


which implies that $t_{n} \rightarrow z(n \rightarrow \infty)$. It follows from (3.22) and (3.25) that

$$
\lim _{n \rightarrow \infty}\left\|t_{n}-x_{n}\right\|=0
$$

Note that $w_{n}-x_{n}=\left(1-\beta_{n}\right)\left(t_{n}-x_{n}\right)$, so by (3.26) and condition (i), we obtain that

$$
\lim _{n \rightarrow \infty}\left\|w_{n}-x_{n}\right\|=0,
$$

which implies that $w_{n} \rightarrow z(n \rightarrow \infty)$. Note that

$$
\left\|w_{n}-W w_{n}\right\| \leq\left\|w_{n}-x_{n}\right\|+\left\|x_{n}-W_{n} w_{n}\right\|+\left\|W_{n} w_{n}-W w_{n}\right\|
$$

Therefore, by (3.13), (3.27), (3.28) and Lemma 2.5, we have

$$
\lim _{n \rightarrow \infty}\left\|w_{n}-W w_{n}\right\|=0
$$

Step $4(z \in F)$. Since $w_{n} \rightarrow z(n \rightarrow \infty)$ and $W$ is nonexpansive, by (3.29), we have

$$
\|z-W z\| \leq\left\|z-w_{n}\right\|+\left\|w_{n}-W w_{n}\right\|+\left\|W w_{n}-W z\right\| \longrightarrow 0 \quad(n \longrightarrow \infty) .
$$

So $z=W z$, that is, $z \in F(W)=\bigcap_{i=1}^{\infty} F\left(T_{i}\right)$.

Next we show that $z \in G$ MEP. Indeed, from (H2) and (1.14), we get

$$
\left\langle B x_{n}, y-u_{n}\right\rangle+\varphi(y)-\varphi\left(u_{n}\right)+\frac{1}{r_{n}}\left\langle y-u_{n}, u_{n}-x_{n}\right\rangle \geq \Theta\left(y, u_{n}\right)
$$

Put $y_{t}=t y+(1-t) z, t \in(0,1]$ and $y \in C$. So $y_{t} \in C$. By (3.31), we have

$$
\begin{aligned}
\left\langle B y_{t}, y_{t}-u_{n}\right\rangle \geq & \left\langle B y_{t}, y_{t}-u_{n}\right\rangle-\left\langle B x_{n}, y_{t}-u_{n}\right\rangle-\varphi\left(y_{t}\right)+\varphi\left(u_{n}\right)-\left\langle y_{t}-u_{n}, \frac{u_{n}-x_{n}}{r_{n}}\right\rangle+\Theta\left(y_{t}, u_{n}\right) \\
= & \left\langle B y_{t}-B u_{n}, y_{t}-u_{n}\right\rangle+\left\langle B u_{n}-B x_{n}, y_{t}-u_{n}\right\rangle \\
& -\varphi\left(y_{t}\right)+\varphi\left(u_{n}\right)-\left\langle y_{t}-u_{n}, \frac{u_{n}-x_{n}}{r_{n}}\right\rangle+\Theta\left(y_{t}, u_{n}\right) \\
\geq & -\left\|B u_{n}-B x_{n}\right\|\left\|y_{t}-u_{n}\right\|-\varphi\left(y_{t}\right)+\varphi\left(u_{n}\right)-\left\|y_{t}-u_{n}\right\|\left\|\frac{u_{n}-x_{n}}{r_{n}}\right\|+\Theta\left(y_{t}, u_{n}\right) .
\end{aligned}
$$

Let $n \rightarrow \infty$ in (3.32), since $B$ is nonexpansive and $\varphi$ is lower semicontinuous, by (3.22), condition (ii) and (H4), we have

$$
\left\langle B y_{t}, y_{t}-z\right\rangle \geq-\varphi\left(y_{t}\right)+\varphi(z)+\Theta\left(y_{t}, z\right)
$$


So, from (H1), (H4), and the above inequality, we obtain

$$
\begin{aligned}
0 & =\Theta\left(y_{t}, y_{t}\right)+\varphi\left(y_{t}\right)-\varphi\left(y_{t}\right) \\
& \leq t \Theta\left(y_{t}, y\right)+(1-t) \Theta\left(y_{t}, z\right)+t \varphi(y)+(1-t) \varphi(z)-\varphi\left(y_{t}\right) \\
& =t\left(\Theta\left(y_{t}, y\right)+\varphi(y)-\varphi\left(y_{t}\right)\right)+(1-t)\left(\Theta\left(y_{t}, z\right)+\varphi(z)-\varphi\left(y_{t}\right)\right) \\
& \leq t\left(\Theta\left(y_{t}, y\right)+\varphi(y)-\varphi\left(y_{t}\right)\right)+(1-t) t\left\langle B y_{t}, y-z\right\rangle,
\end{aligned}
$$

that is,

$$
\Theta\left(y_{t}, y\right)+\varphi(y)-\varphi\left(y_{t}\right)+(1-t)\left\langle B y_{t}, y-z\right\rangle \geq 0
$$

Letting $t \rightarrow 0$ in the above inequality, we obtain for each $y \in C$,

$$
\Theta(z, y)+\varphi(y)-\varphi(z)+\langle B z, y-z\rangle \geq 0
$$

This implies that $z \in G$ MEP.

Finally, we show that $z \in \operatorname{VI}(C, A)$. Define a mapping $T$ as Lemma 2.2. Let $(v, u) \in$ $G(T)$. Since $u-A v \in N_{C} v$ and $t_{n} \in C$, we have $\left\langle v-t_{n}, u-A v\right\rangle \geq 0$. Since $t_{n}=P_{C}\left(u_{n}-\lambda_{n} A y_{n}\right)$, we have

$$
\left\langle v-t_{n}, t_{n}-\left(u_{n}-\lambda_{n} A y_{n}\right)\right\rangle \geq 0,
$$

and hence

$$
\begin{aligned}
\left\langle v-t_{n}, u\right\rangle & \geq\left\langle v-t_{n}, A v\right\rangle \\
& \geq\left\langle v-t_{n}, A v\right\rangle-\left\langle v-t_{n}, \frac{t_{n}-u_{n}}{\lambda_{n}}+A y_{n}\right\rangle \\
& =\left\langle v-t_{n}, A v-A y_{n}-\frac{t_{n}-u_{n}}{\lambda_{n}}\right\rangle \\
& =\left\langle v-t_{n}, A v-A t_{n}\right\rangle+\left\langle v-t_{n}, A t_{n}-A y_{n}\right\rangle-\left\langle v-t_{n} \frac{t_{n}-u_{n}}{\lambda_{n}}\right\rangle \\
& \geq-\left\|v-t_{n}\right\|\left\|A t_{n}-A y_{n}\right\|-\left\|v-t_{n}\right\|\left\|\frac{t_{n}-u_{n}}{\lambda_{n}}\right\| .
\end{aligned}
$$

Since $\lim _{n \rightarrow \infty}\left\|t_{n}-y_{n}\right\|=0$, and $A$ is Lipschitz continuous, by (3.25) and condition (ii), we deduce that $\langle v-z, u\rangle \geq 0$. Since $T$ is maximal monotone, we have $z \in T^{-1} 0$ and so $z \in$ $\operatorname{VI}(C, A)$. Hence $z \in F$.

Step $5\left(z=P_{F} x_{0}\right)$. Put $z^{*}=P_{F} x_{0}$. Since $x_{n}=P_{C_{n}} x_{0}, z \in F$ and the norm is lower semicontinuous, we have

$$
\left\|z^{*}-x_{0}\right\| \leq\left\|z-x_{0}\right\| \leq \liminf _{n \rightarrow \infty}\left\|x_{n}-x_{0}\right\|=\lim _{n \rightarrow \infty}\left\|x_{n}-x_{0}\right\| \leq\left\|z^{*}-x_{0}\right\|,
$$


that is, $\left\|z^{*}-x_{0}\right\|=\left\|z-x_{0}\right\|$. Hence $z=z^{*}=P_{F} x_{0}$, since $z^{*}$ is the unique element in $F$ that minimizes the distance from $x_{0}$.

Thus, $\left\{x_{n}\right\}$ converges strongly to $P_{F} x_{0}$.

Remark 3.2. Theorem 3.1 mainly improves the results of Inchan [10]. To be more precise, Theorem 3.1 improves and extends Theorem 3.1 of [10] from the following several aspects:

(i) from a single nonexpansive mapping to an infinite family of strictly pseudocontractive mappings,

(ii) from generalized equilibrium problems to generalized mixed equilibrium problems,

(iii) from hybrid extragradient methods to hybrid extragradient-like methods,

(iv) the condition of $A$ relaxes to monotone, Lipschitz continuous.

\section{Application}

Theorem 4.1. Let $C$ be a nonempty closed convex subset of a real Hilbert space $H$. Let $\Theta$ be a bifunction from $C \times C$ into $R$ satisfying (H1)-(H4) and $\varphi: C \rightarrow R$ be a lower semicontinuous and convex function with (A1) or (A2). Let $A: C \rightarrow H$ be a monotone, $k$-Lipschitz continuous mapping and $T: C \rightarrow C$ be a $\xi$-strictly pseudocontractive mapping. Let $T_{i}: C \rightarrow C$ be a $\mu_{i}$-strictly pseudocontractive mapping with $F=: \bigcap_{i=1}^{\infty} F\left(T_{i}\right) \cap \mathrm{VI}(C, A) \cap G \mathrm{MEP} \neq \emptyset$ and $\left\{\gamma_{i}\right\}$ be a real sequence such that $0<\gamma_{i} \leq b<1$, for all $i \geq 1$. Let the sequence $\left\{x_{n}\right\}$ be generated $C_{1}=C \subset H, x_{1}=P_{C} x_{0}$,

$$
\begin{gathered}
u_{n} \in C \text { such that } \Theta\left(u_{n}, y\right)+\left\langle(I-T) x_{n}, y-u_{n}\right\rangle \\
+\varphi(y)-\varphi\left(u_{n}\right)+\frac{1}{r_{n}}\left\langle y-u_{n}, u_{n}-x_{n}\right\rangle \geq 0, \quad y \in C, \\
y_{n}=P_{C}\left(u_{n}-\lambda_{n} A u_{n}\right), \\
z_{n}=\alpha_{n} x_{n}+\left(1-\alpha_{n}\right) W_{n}\left(\beta_{n} x_{n}+\left(1-\beta_{n}\right) P_{C}\left(u_{n}-\lambda_{n} A y_{n}\right)\right), \\
C_{n+1}=\left\{z \in C_{n}:\left\|z_{n}-z\right\| \leq\left\|x_{n}-z\right\|\right\}, \\
x_{n+1}=P_{C_{n+1}} x_{0}, \quad \forall n \geq 0 .
\end{gathered}
$$

Assume that the control sequence $\left\{\alpha_{n}\right\},\left\{\beta_{n}\right\} \subset[0,1],\left\{r_{n}\right\} \subset(0,1-\xi)$ and $\left\{\lambda_{n}\right\} \subset(0,1 / 2 k)$ satisfy the following conditions:

(i) $\limsup _{n \rightarrow \infty} \alpha_{n}<1, \limsup _{n \rightarrow \infty} \beta_{n}<1$,

(ii) $0<a \leq \lambda_{n} \leq b<1 / 2 k, 0<d \leq r_{n} \leq e<1-\xi$.

Then $\left\{x_{n}\right\}$ converges strongly to $P_{F} x_{0}$.

Proof. A $\xi$-strictly pseudocontractive mapping is $(1-\xi) / 2$-inverse-strongly monotone. Then taking $s_{n}=1$, for all $n \geq 1$ in Theorem 3.1, we obtain the conclusion.

\section{Acknowledgment}

The authors are extremely grateful to the referees for their useful suggestions that improved the content of the paper. 


\section{References}

[1] P. Kumam, "A hybrid approximation method for equilibrium and fixed point problems for a monotone mapping and a nonexpansive mapping," Nonlinear Analysis, vol. 2, no. 4, pp. 1245-1255, 2008.

[2] G. Cai and C. S. Hu, "A hybrid approximation method for equilibrium and fixed point problems for a family of infinitely nonexpansive mappings and a monotone mapping," Nonlinear Analysis, vol. 3, no. 4, pp. 395-407, 2009.

[3] P. Kumam, "A new hybrid iterative method for solution of equilibrium problems and fixed point problems for an inverse strongly monotone operator and a nonexpansive mapping," Journal of Applied Mathematics and Computing, vol. 29, no. 1-2, pp. 263-280, 2009.

[4] P. L. Combettes and S.A. Hirstoaga, "Equilibrium programming using proximal-like algorithms," Mathematical Programming, vol. 78, no. 1, pp. 29-41, 1997.

[5] J.-W. Peng and J.-C. Yao, "A new hybrid-extragradient method for generalized mixed equilibrium problems, fixed point problems and variational inequality problems," Taiwanese Journal of Mathematics, vol. 12, no. 6, pp. 1401-1432, 2008.

[6] L.-C. Ceng and J.-C. Yao, "A hybrid iterative scheme for mixed equilibrium problems and fixed point problems," Journal of Computational and Applied Mathematics, vol. 214, no. 1, pp. 186-201, 2008.

[7] S. Takahashi and W. Takahashi, "Strong convergence theorem for a generalized equilibrium problem and a nonexpansive mapping in a Hilbert space," Nonlinear Analysis, vol. 69, no. 3, pp. 1025-1033, 2008.

[8] A. Bnouhachem, M. Aslam Noor, and Z. Hao, "Some new extragradient iterative methods for variational inequalities," Nonlinear Analysis, vol. 70, no. 3, pp. 1321-1329, 2009.

[9] L.-C. Ceng, N. Hadjisavvas, and N.-C. Wong, "Strong convergence theorem by a hybrid extragradient-like approximation method for variational inequalities and fixed point problems," Journal of Global Optimization, vol. 46, no. 4, pp. 635-646, 2010.

[10] I. Inchan, "Hybrid extragradient method for general equilibrium problems and fixed point problems in Hilbert space," Nonlinear Analysis, vol. 5, no. 3, pp. 467-478, 2011.

[11] S. Wang, "A general iterative method for obtaining an infinite family of strictly pseudo-contractive mappings in Hilbert spaces," Applied Mathematics Letters, vol. 24, no. 6, pp. 901-907, 2011.

[12] H. Zhou, "Convergence theorems of fixed points for $\kappa$-strict pseudo-contractions in Hilbert spaces," Nonlinear Analysis, vol. 69, no. 2, pp. 456-462, 2008.

[13] R. T. Rockafellar, "On the maximality of sums of nonlinear monotone operators," Transactions of the American Mathematical Society, vol. 149, pp. 75-88, 1970.

[14] K. Shimoji and W. Takahashi, "Strong convergence to common fixed points of infinite nonexpansive mappings and applications," Taiwanese Journal of Mathematics, vol. 5, no. 2, pp. 387-404, 2001.

[15] S.-S. Chang, H. W. Joseph Lee, and C. K. Chan, "A new method for solving equilibrium problem fixed point problem and variational inequality problem with application to optimization," Nonlinear Analysis, vol. 70, no. 9, pp. 3307-3319, 2009. 


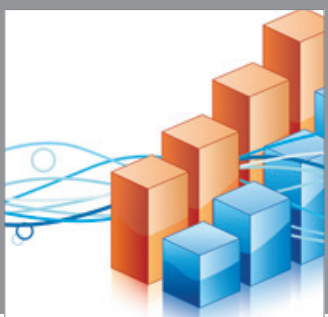

Advances in

Operations Research

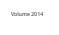

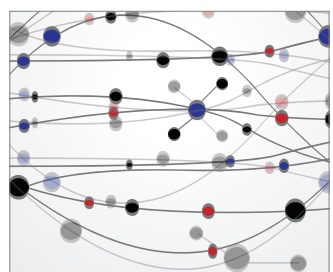

\section{The Scientific} World Journal
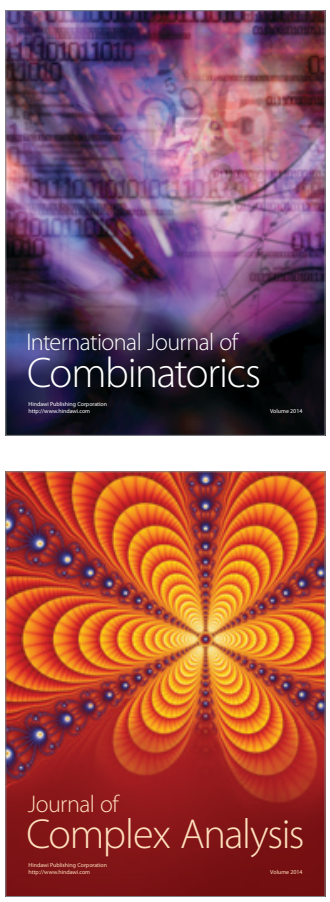

International Journal of

Mathematics and

Mathematical

Sciences
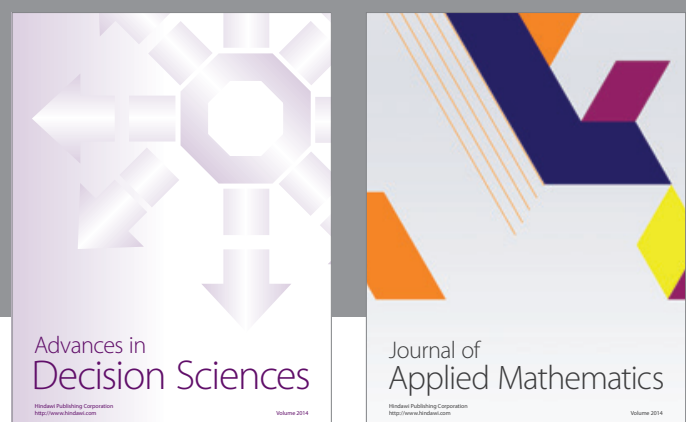

Journal of

Applied Mathematics
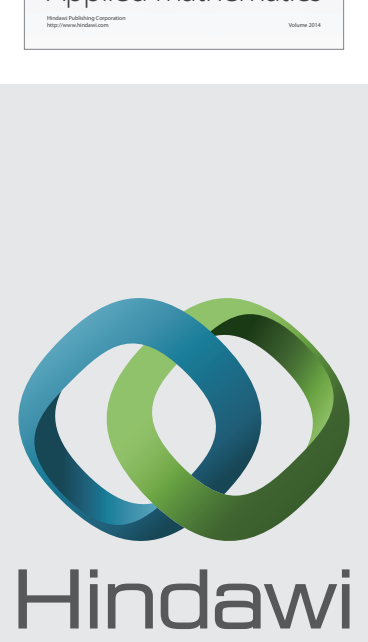

Submit your manuscripts at http://www.hindawi.com
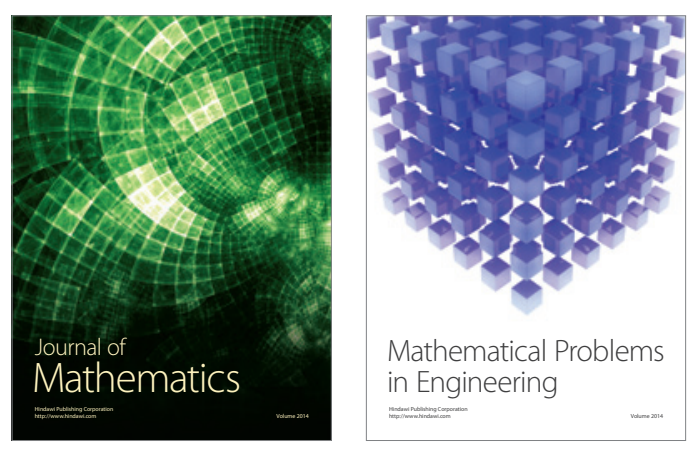

Mathematical Problems in Engineering
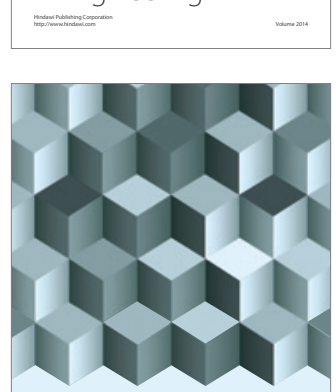

Journal of

Function Spaces
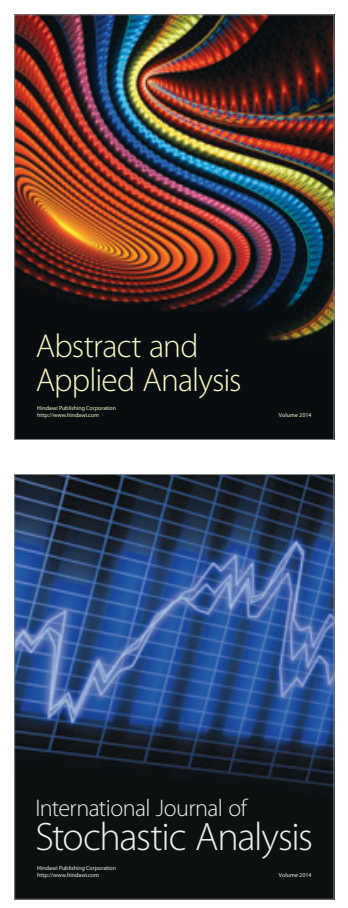

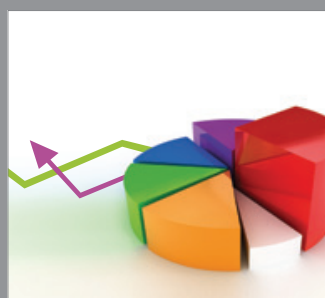

ournal of

Probability and Statistics

Promensencen
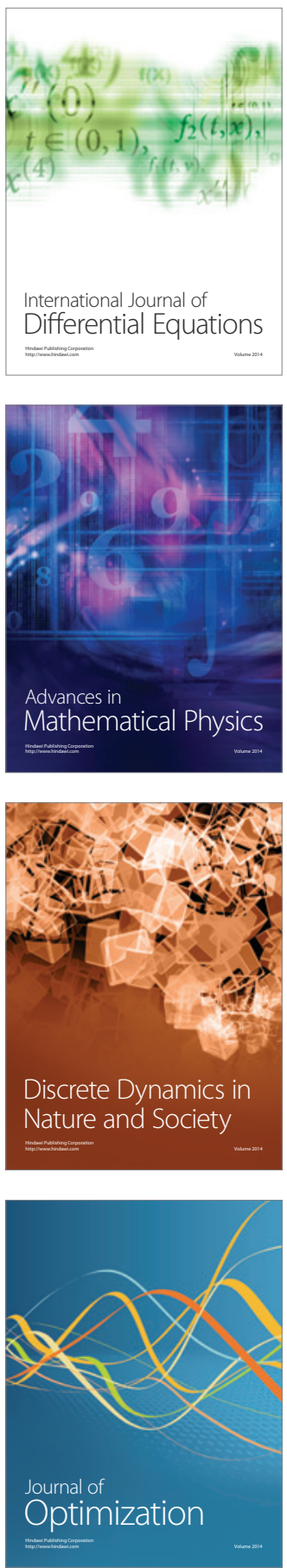